\title{
Desempenho produtivo de leitoas alimentadas com dietas de gestação de baixo ou alto nível de casca de soja
}

\author{
Productive perfomance of gilts fed gestation diets with low or high soybean hulls level
}

\author{
Fabiane Pereira Gentilini ${ }^{1}$ Djane Dallanora ${ }^{2}$ Carlos Henrique Peixoto $^{3}$ \\ Mari Lourdes Bernardi ${ }^{4}$ Ivo Wentz $^{5}$ Fernando Pandolfo Bortolozzo $^{5}$
}

\section{RESUMO}

Leitoas foram alimentadas com dietas de gestação com inclusão de $7 \%$ (T1) e 35\% (T2) de casca de soja, as quais continham níveis de 4,5 e 13,1\% de fibra bruta, respectivamente. Após a inseminação, todas as fêmeas receberam $1,8 \mathrm{~kg}$ da ração T1 (5470Kcal EM dia ${ }^{-1}$ ) até os 35 dias de gestação, quando foram separadas em dois grupos que receberam 2,4kg (7294Kcal EM dia $\left.{ }^{-1}\right)$ da ração $T 1(n=108)$ ou $2,7 \mathrm{~kg}\left(7249 \mathrm{Kcal} \mathrm{EM} \mathrm{dia}^{-1}\right) \mathrm{da}$ ração $T 2$ (n=99). Aos 90 dias de gestação, as fêmeas $T 1$ e T2 receberam respectivamente $3,0 \mathrm{~kg}$ (9117Kcal EM dia $\left.{ }^{-1}\right)$ e 3,4kg (9129Kcal EM dia ${ }^{-1}$ ) de ração. As fêmeas $T 1$ foram mais pesadas que as $T 2$ aos 110 dias de gestação e ao parto $(P<0,05)$. No entanto, o consumo diário médio de ração durante a lactação foi $5,3 \%$ maior para as fêmeas $T 2(P=0,01)$ do que para as fêmeas T1. Não houve efeito dos tratamentos sobre a produção e composição do leite, a variação de peso das fêmeas durante a lactação $e$ o intervalo desmame-estro $(P>0,05)$. O número de nascidos totais, nascidos vivos, natimortos e mumificados foi semelhante $(P>0,05)$ entre os tratamentos. $O$ peso médio ao nascer foi maior $(P=0,03)$ para os leitões das fêmeas $T 1$, mas não houve diferença entre os tratamentos no peso médio da leitegada $(P>0,05)$. $O$ peso e número de leitões ao desmame não foram afetados pelos tratamentos $(P>0,05)$. Os dois níveis de inclusão de casca de soja na dieta de gestação de leitoas resultaram em desempenho produtivo semelhante.

Palavras-chave: casca de soja, gestação, lactação, leitegada, leitoa.

\section{ABSTRACT}

Gilts were fed gestation diets, with inclusion of $7 \%$ (T1) and 35\% (T2) of soybean hulls which had fiber levels of 4.5 and $13.1 \%$, respectively. After insemination, all females received $1.8 \mathrm{~kg}$ of $\mathrm{T1}$ diet $\left(5470 \mathrm{Kcal} \mathrm{ME}\right.$ day $\left.^{-1}\right)$ up to 35 days of gestation, when they were allocated to receive

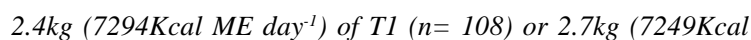
$M E$ day $\left.^{-1}\right)$ of $T 2(n=99)$ diet. At 90 days of gestation $T 1$ and

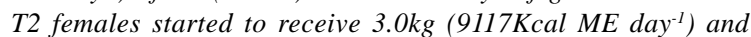

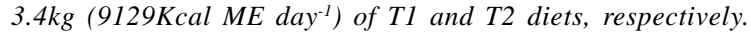
The $T 1$ females were heavier than $T 2$ females, at 110 days of gestation and at parturition $(P<0.05)$. However, the daily average feed intake during lactation was $5.3 \%$ higher for $T 2$ $(P=0.01)$ than for $T 1$ females. There was no effect of treatments on milk production and composition, weight variation during lactation and weaning-to-estrus interval $(P>0.05)$. Treatments were similar for total and born alive litter size, stillborn and mummified $(P>0.05)$. The average birth weight was higher for T1 piglets $(P=0.03)$, but litter weight was similar for treatments $(P>0.05)$. Weaned litter size and weight did not differ for treatments $(P>0.05)$. The two levels of soybean hulls in the gestation diets of gilts resulted in similar performance.

Key words: soybean hulls, gestation, lactation, litter, gilt.

\section{INTRODUÇÃO}

A restrição da oferta de dietas concentradas baseadas em cereais, durante a gestação de fêmeas suínas, visa evitar o excessivo ganho de peso, a possível redução do consumo na lactação, o que diminuiria a produção de leite e aumentaria o intervalo desmame-estro (MEUNIER-SALAÜN et al., 2001). No entanto, a diminuição da saciedade induzida por esta restrição tem efeito negativo no bem-estar das fêmeas (DANIELSEN \& VESTERGAARD, 2001). Por esta razão, tem crescido o interesse em utilizar alimentos volumosos para reprodutoras, especialmente em gestação. Os efeitos da fibra dietética têm sido estudados

${ }^{1}$ Médico Veterinário, Doutora, Rua Emílio Jorge dos Reis, 374, 96020-440, Pelotas, RS. E-mail: fgentilini@bol.com.br. Autor para correspondência.

${ }^{2}$ Médico Veterinário, Mestrando, Faculdade de Veterinária (FAVET), Universidade Federal do Rio Grande do Sul (UFRGS), Av. Bento Gonçalves, 9090, 91540-000, Porto Alegre, RS.

${ }^{3}$ Médico Veterinário, Mestre, FAVET, UFRGS.

${ }^{4}$ Médico Veterinário, Doutor, Professor Adjunto, Faculdade de Agronomia, UFRGS.

${ }^{5}$ Médico Veterinário, Doutor, Professor Adjunto, FAVET, UFRGS. 
quanto aos aspectos nutricionais e metabólicos, e suas conseqüências no desempenho produtivo e reprodutivo (RAMONET et al., 1999).

A casca de soja, um subproduto obtido da industrialização da soja para extração do óleo, é uma película que reveste o grão, e que tem sido utilizada na alimentação animal. Consiste de uma fonte rica em fibra de baixa lignificação, com um teor de proteína bruta de cerca de $12 \%$ e um teor de fibra bruta ao redor de $36 \%$ (BLAS et al., 1999), constituindo 8\% do peso da semente (KLOPFENSTEIN \& OWEN, 1987).

KORNEGAY (1981) utilizou a casca de soja como fonte de fibra na dieta de suínos em crescimentoterminação e de porcas em gestação, e constatou que as fêmeas gestantes apresentaram uma melhor digestibilidade dos nutrientes da ração, com um melhor aproveitamento da fibra dietética, em comparação aos suínos em crescimento-terminação. Animais adultos apresentam seu trato gastrintestinal mais desenvolvido, o que propicia a atuação da microbiota do intestino grosso (cólon e ceco) sobre a fibra, produzindo ácidos graxos voláteis que são absorvidos e utilizados como fonte de energia por estes animais (NOBLET \& SHI, 1993).

O objetivo deste estudo foi avaliar o efeito da inclusão de dois níveis de casca de soja, na dieta de leitoas em gestação, sobre o ganho de peso durante a gestação, o consumo de ração e a perda de peso durante a lactação, o número total de leitões nascidos, nascidos vivos, natimortos e mumificados, peso ao nascer dos leitões, além do intervalo desmame-estro das fêmeas. Avaliou-se, ainda, o efeito dos tratamentos sobre a composição e produção de leite das fêmeas, o número e peso dos leitões ao desmame.

\section{MATERIAL E MÉTODOS}

O estudo foi realizado em uma unidade produtora de leitões, localizada no oeste do estado de Santa Catarina, no período de janeiro a julho de 2001. Foram selecionadas leitoas da linhagem Cambourough $22^{\circledR}$, com peso e idade médios de $135,1 \mathrm{~kg}$ e 205 dias. As fêmeas foram inseminadas no terceiro estro (idade média de 220 dias), com doses de sêmen contendo três bilhões de espermatozóides, em intervalos médios de 12h. O diagnóstico de estro foi realizado duas vezes por dia, na presença do macho. Após a detecção do estro, as fêmeas foram imediatamente inseminadas, recebendo uma segunda e terceira doses em intervalos médios de 12 e $24 \mathrm{~h}$, da primeira.

Da inseminação até os 34 dias de gestação todas as fêmeas receberam uma dieta com inclusão de $7 \%$ de casca de soja (4,5\% de fibra bruta), na quantida- de de $1,8 \mathrm{~kg}$ de ração/dia (5470Kcal EM dia $\left.{ }^{-1}\right)$. Aos 35 dias de gestação, 207 fêmeas foram distribuídas em dois grupos que passaram a receber rações com diferentes níveis de fibra bruta. A partir deste momento, a quantidade de ração oferecida foi ajustada, segundo o tipo de dieta, de modo que a ingestão de energia fosse semelhante para os dois grupos de fêmeas. As fêmeas $\mathrm{T} 1(\mathrm{n}=108)$ continuaram a receber a ração com $4,5 \%$ de fibra bruta na quantidade de $2,4 \mathrm{~kg}$ de ração/dia $\left(7294 \mathrm{Kcal} \mathrm{EM} \mathrm{dia}^{-1}\right)$. As fêmeas T2 $(\mathrm{n}=99)$ receberam a ração com inclusão de $35 \%$ de casca de soja $(13,1 \%$ de fibra bruta), na quantidade de $2,7 \mathrm{~kg}$ de ração/dia (7249Kcal EM dia-1 $)$. A partir dos 90 dias de gestação, as fêmeas T1 e T2 passaram a receber 3,0 e 3,4kg de ração/dia (9117 e 9129Kcal EM dia ${ }^{-1}$, respectivamente). Ambas as rações eram do tipo farelada e foram formuladas com base no NRC (1998), sendo a composição percentual das mesmas apresentada na Tabela 1. Os arraçoamentos foram realizados às 6 e às $14 \mathrm{~h}$, com ração seca, e toda a ração oferecida foi consumida pelas fêmeas. A água foi fornecida à vontade.

Aos 110 dias de gestação, as fêmeas foram transferidas para as salas de maternidade, onde perma-

Tabela 1 - Composição percentual das dietas de gestação de leitoas.

\begin{tabular}{|c|c|c|}
\hline \multirow[b]{2}{*}{ Ingredientes $(\mathrm{kg})$} & \multicolumn{2}{|c|}{ Dietas experimentais } \\
\hline & $\mathrm{T} 1$ & $\mathrm{~T} 2$ \\
\hline Milho & 71,71 & 47,00 \\
\hline Farelo de soja, $47 \%$ & 17,23 & 14,18 \\
\hline Casca de soja & 7,00 & 35,00 \\
\hline Calcário & 1,41 & 1,39 \\
\hline Foscálcio, $45 \%$ & 1,75 & 1,53 \\
\hline Sal & 0,50 & 0,50 \\
\hline Premix Minerais $^{1}$ e Vitaminas ${ }^{2}$ & 0,40 & 0,40 \\
\hline \multicolumn{3}{|l|}{ Valores calculados } \\
\hline Proteína bruta (\%) & 14,50 & 14,50 \\
\hline Fibra bruta $(\%)$ & 4,50 & 13,08 \\
\hline Cálcio (\%) & 0,99 & 0,99 \\
\hline Fósforo total (\%) & 0,66 & 0,70 \\
\hline Fósforo disponível (\%) & 0,42 & 0,40 \\
\hline Lisina $(\%)$ & 0,73 & 0,78 \\
\hline Metionina-cistina (\%) & 0,52 & 0,50 \\
\hline Triptofano (\%) & 0,18 & 0,18 \\
\hline Treonina $(\%)$ & 0,54 & 0,55 \\
\hline $\begin{array}{l}\text { Energia metabolizável } \\
\text { (Kcal/kg) }\end{array}$ & 3039 & 2685 \\
\hline
\end{tabular}

${ }^{1}$ Composição (1kg/tonelada de ração): 124.999,8mg Fe; $15.000,0 \mathrm{mg} \mathrm{Cu} ; 59.819,7 \mathrm{mg} \mathrm{Mn} ; 9.999,9 \mathrm{mg} \mathrm{Zn} ; 1.000 \mathrm{mg}$ iodo; 400mg Se; $20.000 \mathrm{mg}$ BHT.

${ }^{2}$ Composição (500g/tonelada de ração): $10.000 .000 \mathrm{UI}$ vit. A; 2.000.000UI vit. D3; $20.000 \mathrm{UI}$ vit. E; $2.000 \mathrm{UI}$ vit. K; $4.000 \mathrm{UI}$ vit.

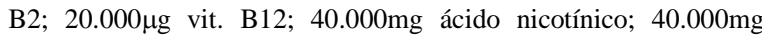
ácido pantotênico. 
neceram em média até $19^{\circ}$ dia de lactação. Até o terceiro dia pós-parto, as fêmeas continuaram a receber as rações T1 e T2 de gestação e, a partir deste momento, todas passaram a receber ração de lactação, com níveis protéico e energético de $16,8 \%$ e $3.298,38 \mathrm{Kcal} \mathrm{kg}^{-1}$, respectivamente. Durante o período de aleitamento, a ração foi fornecida na forma farelada seca, às 5:00, 8:00, 15:30 e 17:00h, em quantidades próximas de $1,5 \mathrm{~kg}$ a cada arraçoamento. As fêmeas dispunham de livre acesso à água.

Uma semana após o início do experimento (42 dias de gestação), um grupo de fêmeas (T1= 56 e $\mathrm{T} 2=48$ ) foi pesado, não tendo sido verificadas diferenças entre os tratamentos $(\mathrm{P}>0,05 ; \mathrm{T} 1=165,35 \mathrm{~kg}$ vs. $\mathrm{T} 2=$ $165,09 \mathrm{~kg}$ ), evidenciando a homogeneidade dos grupos. Grupos de fêmeas de cada tratamento foram pesadas $\operatorname{aos} 70(\mathrm{~T} 1=47$ e T2=39) e $110(\mathrm{~T} 1=101$ e T2=94) dias de gestação. Após o parto, foram pesadas 91 fêmeas de cada tratamento.

Ao nascimento, foi avaliado o número total de leitões nascidos, nascidos vivos, natimortos e mumificados. Os leitões foram pesados individualmente ao nascer e as leitegadas foram padronizadas em até $48 \mathrm{~h}$ pós-parto. A padronização das leitegadas foi feita entre fêmeas do mesmo tratamento, as quais permaneceram, em média, com 10 leitões/leitegada, de modo a evitar que um número desigual de leitões pudesse alterar as respostas de desempenho, tanto das fêmeas como dos leitões. No entanto, não foi possível padronizar o tamanho da leitegada em todas as fêmeas de cada tratamento, em virtude da diferença de datas de parto e/ou pelo número insuficiente de leitões. Assim, as fêmeas que ficaram com menor número de leitões não foram usadas para as avaliações efetuadas durante o período de lactação, mas os dados de todas as fêmeas que pariram foram usados nas análises de nascidos totais, nascidos vivos, natimortos e mumificados. Durante todo o período de aleitamento os leitões não receberam ração, de modo a efetuar uma avaliação do desempenho dos leitões em função do real potencial da fêmea e não de uma possível compensação pelo consumo de ração.

$\mathrm{Na}$ lactação, avaliou-se a perda de peso das fêmeas, do parto ao desmame (T1=67 e T2=63), o consumo diário médio de ração (CDMR), a composição do leite e a produção diária média de leite (PDML). O CDMR foi medido em 114 leitoas $(\mathrm{T} 1=56 \mathrm{e}$ T2=58). A cada dois dias, um saco contendo uma quantidade fixa de ração era colocado em frente à cela parideira de cada fêmea, a partir do qual eram retiradas as quantidades fornecidas em cada arraçoamento. Por ocasião da reposição de ração eram pesadas as sobras que restaram no saco e, assim, calculada a quantidade ingerida naquele período, por cada fêmea. Após o desmame, avaliou-se também o intervalo desmame-estro (IDE). Com relação às leitegadas, avaliou-se o número de leitões desmamados e o peso médio dos leitões ao desmame.

Para análise da composição do leite utilizou-se 64 leitoas (T1=34 e T2=30), com média de 10 leitões/leitegada ( 9 a 11 leitões/leitegada). A coleta de amostras de $250 \mathrm{~mL}$ de leite foi realizada através da ordenha manual, no $10^{\circ}$ dia de lactação, após aplicação intramuscular de $1,5 \mathrm{~mL}$ de ocitocina. As amostras foram armazenadas $\mathrm{a}-20^{\circ} \mathrm{C}$ para análise posterior. Foram determinadas a densidade, matéria seca total, gordura, lactose e proteína pelo uso de termolactodensímetro, processo indireto de Fleischmann, métodos de Gerber, de Felhing e micro-Kjeldahl, respectivamente, descritos pelo INSTITUTO ADOLFO LUTZ (1985).

Para medir a produção diária média de leite (PDML) utilizaram-se 49 fêmeas (T1=25 e T2=24). A estimativa da produção leiteira das porcas foi feita no $14^{\circ}$ dia de lactação através do método descrito por LEWIS et al. (1978), de pesagem da leitegada imediatamente antes e após a mamada, sendo a diferença de peso observada considerada como a quantidade de leite (em gramas) obtida pelos leitões e, portanto, a produção por mamada. $\mathrm{O}$ procedimento teve início com a separação da fêmea e sua leitegada, no interior da gaiola parideira, não permitindo o contato físico entre a porca e a sua leitegada. No entanto, os leitões dispunham de livre acesso à água e ao escamoteador. Passados 55 a 60 minutos, a leitegada foi pesada e colocada para mamar. No momento em que os dois primeiros leitões afastaram-se da glândula mamária, ao redor de 2 a 3 minutos após o início da mamada, toda a leitegada foi retirada e pesada novamente. Foram acrescentados 5 e $10 \mathrm{~g}$ por leitão que defecou ou urinou, respectivamente, durante o período da mamada. Este procedimento foi repetido seis vezes em intervalos de 55 a 60 minutos. Das seis medidas realizadas, as duas primeiras foram descartadas por serem consideradas adaptativas para a fêmea e sua leitegada. Portanto, para o cálculo da produção diária de leite da fêmea, utilizou-se a média das quatro últimas medidas, sendo multiplicada por 24, estimando-se assim a produção diária de leite.

Os dados foram analisados pelo procedimento GLM (SAS, 1999) sendo as médias comparadas pelo teste t, com um nível de significância de 5\%. As variáveis ganho de peso durante a gestação, perda de peso durante a lactação, número total de leitões nascidos, nascidos vivos, composição e produção de leite, número de leitões desmamados e intervalo desmameestro foram analisadas conforme o seguinte modelo 
estatístico:

$$
\mathrm{Y}_{\mathrm{ij}}=\mu+\mathrm{CS}_{\mathrm{i}}+\mathrm{e}_{\mathrm{ij}}
$$

onde,

$\mathrm{Y}_{\mathrm{ij}}=$ observação realizada em cada fêmea,

leitão ou amostra de leite;

$\mu=$ média geral;

$\mathrm{CS}_{\mathrm{i}}=$ efeito do nível de inclusão de casca de

soja i;

observação.

$\mathrm{e}_{\mathrm{ij}}=$ erro experimental $\mathrm{j}$ associado com $\mathrm{a}$

Neste mesmo modelo, foi incluída a duração da lactação como covariável para a análise do consumo de ração durante a lactação e do peso médio dos leitões ao desmame. Para a variável peso ao nascer, o número total de nascidos foi incluído como covariável. Os números de natimortos e de mumificados foram submetidos à análise não-paramétrica pelo procedimento NPAR1WAY (SAS, 1999) sendo os rankings médios comparados pelo teste de Wilcoxon-Mann-Whitney. As taxas de parto ajustadas foram comparadas pelo teste Qui-quadrado.

\section{RESULTADOS E DISCUSSÃO}

Das 207 fêmeas submetidas aos tratamentos, a partir dos 35 dias de gestação, não houve diferença na taxa de parto ajustada $(\mathrm{P}>0,05)$ entre as dietas baixa (T1) e alta (T2) fibra (Tabela 2). Após o início dos tratamentos, algumas fêmeas retornaram ao estro $(n=6)$, abortaram $(n=4)$ ou morreram $(n=2)$, não sendo, portanto, analisadas em termos de desempenho.

Não houve diferença $(\mathrm{P}>0,05)$ no peso aos 70 dias de gestação entre as fêmeas T1 e T2 (Tabela 2). As fêmeas T1 pesaram mais aos 110 dias de gestação $(\mathrm{P}=0,05)$, quando comparadas às $\mathrm{T} 2$. Contudo, durante a lactação, verificou-se que as fêmeas T2 consumiram $5,3 \%$ (310g/dia, em média) a mais de ração $(\mathrm{P}=0,01)$, do que as $\mathrm{T} 1$, semelhante à diferença observada por MATTE et al. (1994), entre dietas com alto e baixo teor de fibra. Mesmo recebendo dietas com mesma quantidade de energia metabolizável (EM), no presente estudo, o aumento da taxa de passagem do alimento pelo trato gastrintestinal, diminuindo a digestibilidade dos demais nutrientes da dieta, principalmente da energia (POLLMANN et al., 1979), provavelmente seja a razão do menor ganho de peso das fêmeas que ingeriram maior quantidade de fibra.

Como o consumo alimentar durante a fase de aleitamento é mais comprometido em primíparas, é de grande interesse que, durante a lactação, o volume estomacal desta categoria possa ser aumentado, o que fez com que vários autores adicionassem alimentos fibrosos à dieta destas fêmeas, no período gestacional (POLLMANN et al., 1979; NUZBACK et al., 1984;

Tabela 2 - Efeito do nível de fibra da dieta de gestação sobre o desempenho produtivo e reprodutivo de leitoas e de suas leitegadas (médias \pm desviopadrão).

\begin{tabular}{|c|c|c|c|c|c|}
\hline \multirow[b]{2}{*}{ Parâmetros } & \multicolumn{4}{|c|}{ Teor de fibra na dieta } & \multirow[b]{2}{*}{$\mathrm{P}$} \\
\hline & $\mathrm{n}$ & $\mathrm{T} 1(4,5 \%)$ & $\mathrm{n}$ & $\mathrm{T} 2(13,1 \%)$ & \\
\hline \multicolumn{6}{|l|}{ Fêmeas } \\
\hline Peso médio aos 70 dias de gestação (kg) & 47 & $189,8 \pm 12,0$ & 39 & $190,3 \pm 12,0$ & 0,85 \\
\hline Peso médio aos 110 dias de gestação (kg) & 101 & $214,7^{\mathrm{a}} \pm 15,3$ & 94 & $210,4^{\mathrm{b}} \pm 15,2$ & 0,05 \\
\hline Taxa de parto ajustada (\%) & 108 & 93,5 & 97 & 96,9 & 0,26 \\
\hline Peso médio após o parto (kg) & 91 & $195,8^{\mathrm{a}} \pm 15,1$ & 91 & $190,5^{\mathrm{b}} \pm 14,7$ & 0,02 \\
\hline Consumo diário de ração na lactação (kg) & 56 & $5,9^{\mathrm{a}} \pm 0,6$ & 58 & $6,2^{\mathrm{b}} \pm 0,8$ & 0,01 \\
\hline Perda de peso da fêmea na lactação (kg) & 67 & $7,2 \pm 9,1$ & 63 & $5,9 \pm 9,3$ & 0,43 \\
\hline Peso da fêmea ao desmame (kg) & 67 & $189,7 \pm 14,9$ & 63 & $187,1 \pm 13,6$ & 0,30 \\
\hline Intervalo desmame-estro (dias) & 84 & $7,85 \pm 5,98$ & 84 & $8,98 \pm 8,42$ & 0,32 \\
\hline \multicolumn{6}{|l|}{ Leitões } \\
\hline Nascidos totais & 101 & $10,77 \pm 2,47$ & 94 & $11,35 \pm 2,45$ & 0,16 \\
\hline Nascidos vivos & 101 & $9,97 \pm 2,45$ & 94 & $10,65 \pm 2,41$ & 0,09 \\
\hline Natimortos & 101 & $0,60 \pm 0,92$ & 94 & $0,49 \pm 0,76$ & $*$ \\
\hline Mumificados & 101 & $0,19 \pm 0,50$ & 94 & $0,21 \pm 0,47$ & $*$ \\
\hline Peso médio dos leitões ao nascer (kg) & 101 & $1,44^{\mathrm{a}} \pm 0,21$ & 94 & $1,36^{\mathrm{b}} \pm 0,23$ & 0,03 \\
\hline Peso médio da leitegada ao nascer (kg) & 101 & $15,53 \pm 2,87$ & 94 & $15,39 \pm 2,73$ & 0,63 \\
\hline Leitões desmamados & 84 & $9,21 \pm 1,50$ & 84 & $8,95 \pm 2,04$ & 0,36 \\
\hline Peso médio dos leitões ao desmame (kg) & 84 & $4,92 \pm 1,18$ & 84 & $4,89 \pm 1,09$ & 0,76 \\
\hline
\end{tabular}

${ }^{\mathbf{a}, \mathbf{b}}$ Médias com letras distintas, na mesma linha, diferem pelo teste $\mathrm{t}$.

* Não houve diferença entre os tratamentos $(\mathrm{P}>0,05)$, quando os rankings médios foram comparados pelo teste de Wilcoxon-Mann-Whitney. 
POND et al., 1985; YAN et al., 1995; EWAN et al., 1996). Em várias espécies, incluindo suínos, o volume, o peso e a capacidade do trato digestivo aumentam com a elevação da porcentagem de fibra na ração (COEY \& ROBINSON, 1954; HANSEN et al., 1992), sugerindo que o fornecimento de uma ração rica em fibra durante a gestação pode levar a um aumento do estômago e, conseqüentemente, a um maior consumo durante a subsequiente fase de lactação.

MATTE et al. (1994), trabalhando com três níveis de FB na dieta de gestação $(2,2 ; 10,1$ e 20,4mg $\mathrm{kg}^{-1}$ de matéria seca, respectivamente), durante dois ciclos reprodutivos consecutivos, constataram que as fêmeas que receberam a dieta altamente fibrosa $(20,4 \mathrm{mg}$ $\mathrm{kg}^{-1}$ ) ganharam menos peso durante a gestação e consumiram 5\% a mais durante a lactação. Outros autores (FARMER et al., 1996; DANIELSEN \& VESTERGAARD, 2001) também observaram que as fêmeas que receberam dieta rica em fibra, durante a gestação, consumiram mais durante a lactação.

Não foram observadas diferenças entre os tratamentos nas variáveis perda de peso da fêmea na lactação e IDE $(\mathrm{P}>0,05)$. Resultados semelhantes foram obtidos por VESTERGAARD \& DANIELSEN (1998) quando compararam duas dietas ricas em fibra, de diferentes fontes (polpa de beterraba e mistura de alimentos fibrosos) com a dieta padrão (milho e farelo de soja).

Os tratamentos não afetaram o total de leitões nascidos, nascidos vivos, natimortos e mumificados ( $\mathrm{P}>0,05)$. REESE (1997) sugere que o aumento do nível de fibra na dieta de gestação levaria ao aumento do tamanho da leitegada, o que foi constatado por CARTER et al. (1987). No entanto, outros autores (MATTE et al., 1994; VESTERGAARD \& DANIELSEN, 1998; DANIELSEN \& VESTERGAARD, 2001) não verificaram efeito de dietas com diferentes teores e fontes de fibra sobre o tamanho da leitegada.

O peso médio dos leitões, ao nascerem, diferiu entre os tratamentos, tendo sido menor para os leitões nascidos das fêmeas T2 $(\mathrm{P}=0,03)$. Quando considerado o peso médio da leitegada, no entanto, não houve diferença entre os tratamentos $(\mathrm{P}>0,05)$. DANIELSEN \& VESTERGAARD (2001) observaram que fêmeas submetidas à dieta rica em polpa de beterraba (446g fibra dietética $\mathrm{kg}^{-1}$ ) produziram leitões mais leves, quando comparadas às fêmeas que receberam dietas controle ( $176 \mathrm{~g}$ fibra dietética $\mathrm{kg}^{-1}$ ) ou uma mistura de alimentos fibrosos ( $344 \mathrm{~g}$ fibra dietética $\mathrm{kg}^{-1}$ ). O provável menor aporte energético, causado pela maior inclusão de fibra, o que reduz a digestibilidade da energia e outros nutrientes da dieta (KORNEGAY, 1981), deve ter sido o fator responsável pelo menor peso ao nascer dos leitões nascidos de fêmeas submetidas à dieta rica em fibra. Tem sido mostrado que o peso médio ao nascer dos leitões depende dos níveis energéticos na gestação (HENRY \& ETIENNE, 1978). Para um dado nível alimentar, o peso ao nascer dos leitões diminuiu quando a porcentagem de feno de alfafa aumentou de 0 para $95 \%$ na dieta de gestação (CALVERT et al., 1985). Segundo MEUNIER-SALAÜN et al. (2001), quando dietas ricas em fibra são usadas, o tamanho da leitegada e o peso ao nascer dos leitões podem variar em função da fonte de fibra ou da sua taxa de inclusão na dieta de gestação.

Apesar do menor peso ao nascer para os leitões oriundos das fêmeas T2, o nível de fibra não afetou o peso médio dos leitões ao desmame $(\mathrm{P}>0,05)$, confirmando observações de outros autores (POND et al., 1985; DANIELSEN \& VESTERGAARD, 2001). O maior consumo de ração das fêmeas T2, juntamente com a mobilização de reservas, durante a lactação, foram suficientes para garantir o crescimento dos leitões e compensar seu menor peso ao nascer, semelhante ao verificado por MATTE et al. (1994).

A produção de leite no suíno é bastante variável, sendo que a ordem de parição, o número de leitões mamando e/ou nascidos, o nível nutricional, a raça, o estágio de lactação, a estação do ano, o ambiente social e o fotoperíodo são alguns dos fatores responsáveis por esta variação (SPEER \& COX, 1984). No presente estudo, a composição do leite, no $10^{\circ}$ dia de lactação, não foi afetada pelo nível de inclusão de casca de soja na ração de gestação $(\mathrm{P}>0,05)$. Não se observou diferença $(\mathrm{P}>0,05)$ na produção diária de leite, no $14^{\circ}$ dia de lactação (tabela 3), entre as fêmeas dos dois tratamentos. FARMER et al. (1996) também não observaram diferença na produção de leite, no $29^{\circ}$ dia de lactação, de leitoas submetidas a dietas de gestação com dois níveis distintos de FB (4,1 e 15,3\%).

Segundo MATTE et al. (1994), as exigências de nutrientes não tão elevadas por parte das fêmeas gestantes tornam o uso de dietas com ingredientes fibrosos de baixo custo possível e economicamente atrativo, ao mesmo tempo que minimiza os possíveis efeitos adversos da restrição do volume de alimento oferecido no período gestacional. O custo dos ingredientes utilizados na ração variam de uma época para outra, além de sua disponibilidade ser variável de uma região para outra, o que implica em possíveis oscilações do custo final da ração. No presente estudo, os dados de desempenho de um ciclo produtivo das fêmeas não foram favoráveis a nenhum dos níveis de casca de soja utilizados, indicando que tanto o nível baixo como o elevado poderiam ser usados.

A decisão de usar ou não dietas de gestação com alto teor de fibra deve, no entanto, além dos 
Tabela 3 - Composição e produção de leite (média \pm desvio-padrão) de fêmeas suínas submetidas a dietas de gestação com baixo (T1) e alto (T2) teor de fibra bruta.

\begin{tabular}{llllll}
\hline & \multicolumn{3}{c}{ Teor de fibra bruta na dieta } \\
\cline { 2 - 5 } Itens & $\mathrm{n}$ & $\mathrm{T} 1(4,5 \%)$ & $\mathrm{n}$ & $\mathrm{T} 2(13,1 \%)$ \\
\hline Produção de leite no $14^{\mathrm{o}} \mathrm{dia}(\mathrm{kg})$ & 25 & $5,09 \pm 2,22$ & 24 & $4,99 \pm 2,38$ \\
Composição do leite no $10^{\circ} \mathrm{dia}$ & & & 30 & $1033,54 \pm 1,67$ & 0,88 \\
Densidade & 34 & $1032,99 \pm 2,36$ & 30 & $20,15 \pm 1,47$ \\
Matéria seca total (\%) & 34 & $20,06 \pm 1,46$ & 30 & $9,57 \pm 1,25$ & 0,81 \\
Gordura (\%) & 34 & $9,68 \pm 1,49$ & 30 & $4,53 \pm 0,36$ & 0,75 \\
Lactose (\%) & 34 & $4,53 \pm 0,27$ & 30 & $5,09 \pm 0,50$ \\
Proteína (\%) & 34 & $4,92 \pm 0,26$ & & 0,93 \\
\hline
\end{tabular}

aspectos relativos ao desempenho, considerar também os possíveis efeitos benéficos no aumento da saciedade, na redução da incidência de comportamentos esteriotipados e consequente melhoria do bemestar das fêmeas, conforme observado por vários autores (ROBERT et al., 1993; RAMONET et al., 1999; MEUNIER-SALAÜN et al., 2001). Segundo MEUNIER-SALAÜN et al. (2001), o uso de dietas com alto teor de fibra pode ser limitado pelo baixo valor nutricional de alguns ingredientes fibrosos, a dificuldade no manuseio e armazenamento de rações mais volumosas e o maior volume de dejetos sólidos produzidos. Estes aspectos evidenciam a necessidade de maiores informações que permitam adequar a formulação e a quantidade fornecida de dietas ricas em fibra para um aumento do bem-estar animal ao mesmo tempo que satisfaça as exigências ambientais e econômicas.

\section{CONCLUSÃO}

É possível usar casca de soja até $35 \%$, correspondendo a $13 \%$ de fibra bruta, na dieta de gestação de leitoas, sem afetar o seu desempenho durante um ciclo produtivo.

\section{REFERÊNCIAS BIBLIOGRÁFICAS}

BLAS, C.; MATEOS, G.G.; REBOLLAR, P.G. Cascarina de Soja. Normas FEDNA para la formulación de piensos compuestos. Madrid : Fundación Española para el desarrollo de la nutrición animal. Departamento de Producción Animal, Universidad Politécnica de Madrid, 1999. p.299-302.

CALVERT, C.C.; STEELE, N.C.; ROSEBROUGH, R.W. Digestibility of fiber components and reproductive performance of sows fed high levels of alfalfa meal. Journal of Animal Science, v.61, p.595-602, 1985.

CARTER, D.I. et al. The effect of fiber intake by gravid swine during three consecutive parities on sow and litter performance. Journal of Animal Science, v.65 (supplement 1), p.89, 1987. (abstract).
COEY, W.E.; ROBINSON, K.L. Some effects of dietary crude fiber on live weight and carcass conformation of pigs. Journal of Agriculture Science, v.45, p.41, 1954.

DANIELSEN, V.; VESTERGAARD, E-M. Dietary fibre for pregnancy sows: effect on performance and behaviour. Animal Feed Science and Technology, v.90, p.71-80, 2001.

EWAN, R. et al. Effect of addition of fiber to gestation diets on reproductive performance of sows. Journal of Animal Science. v.74 (supplement 1), p.190, 1996.

FARMER, C.; ROBERT, S.; MATTE, J.J. Lactation performance of sows fed a bulky diet during gestation and receiving growth hormone-releasing factor during lactation. Journal of Animal Science, v.74, p.1298-1306, 1996.

HANSEN, I.K.; KNUDSEN, E.B.; EGGUM, B.O. Gastrointestinal implications in the rat of wheat bran, oats bran and pea fiber. British Journal of Nutrition, v.68, p.51, 1992.

HENRY, Y.; ETIENNE, M. Alimentation énergétique du porc. Journées de la Recherche Porcine en France, v.10, p.119$165,1978$.

INSTITUTO ADOLFO LUTZ. Normas Analíticas do Instituto Adolfo Lutz. In: _-___. Métodos químicos e físicos para análise de alimentos. 3.ed. São Paulo, 1985. $533 \mathrm{p}$.

KLOPFENSTEIN, T.; OWEN, F. Soybean hulls - an energy supplement for ruminants. Animal Health \& Nutrition, abril, p.28-32, 1987.

KORNEGAY, E.T. Soybean hulls digestibility by sows and feeding value for growing-finishing swine. Journal of Animal Science. v.53, p.138-145, 1981.

LEWIS, A.J.; SPEER, V.C.; HAUGHT, D.G. Relationship between yield and composition of sows' milk and weight gains of nursing pigs. Journal of Animal Science, v.47, p.634, 1978.

MATTE, J.J. et al. Effect of bulky diets based on wheat bran or oat hulls on reproductive performance of sows during their first two parities. Journal of Animal Science, v.72, p.1754$1760,1994$.

MEUNIER-SALAÜN, M.C.; EDWARDS, S.A.; ROBERT, S. Effect of dietary fibre on the behaviour and health of restricted 
fed sow. Animal Feed Science and Technology, v.90, p.5369,2001

NOBLET, J.; SHI, X.S. Comparative digestibility of energy and nutrients in growing pigs fed ad libitum and adult sows fed at maintenance. Livestock Production Science, v.34, p.137-152, 1993.

NRC (NATIONAL RESERCH COUNCIL) Committee on Animal Nutrition. Subcommittee on Swine Nutrition. Washington : NUTRIENT REQUERIMENTS OF SWINE. Washington : National Academy of Science, 1998. 189p.

NUZBACK, L.J.; POLLMANN, D.S.; BEHNKE, K.C. Effect of particle size and physical form of sun-cured alfafa on digestibility for gravid swine. Journal of Animal Science, v.58, p.378-385, 1984.

POLLMANN, D.S.; DANIELSON, D. M.; PEO Jr., E.R. Value of high fiber diets for gravid swine. Journal of Animal Science, v.48, p.1385-1393, 1979.

POND, W.G.; YEN, J.T.; VAREL, V.H. Effects of level and source of dietary fiber in gestation on reproduction performance and nutrient digestibility in gilts. Nutrition Reports International, v.32, p.505-514, 1985.

RAMONET, Y.; MEUNIER-SALAÜN, M.C.; DOURMAD, J.Y. High-fibre diets in pregnant sows: digestive utilization and effects on the behavior of the animals. Journal of Animal Science, v.77, p.591-599, 1999.

REESE, D.E. Dietary fiber in sows gestation diets reviewed. Feedstuffs Nutrition and Health/Swine, v.23, p.11-15, 1997.

ROBERT, S. et al. High-fibre diets for sows: effects on stereotypies and adjunctive drinking. Applied Animal Behavior Science, v.37, p.297-309, 1993.

SAS INSTITUTE. SAS/STAT User's guide. Version 8. Cary, 1999. 1464p.

SPEER, V.C.; COX, D.F. Estimating milk yield of sows. Journal of Animal Science, v.59, p.1281-1285, 1984.

VESTERGAARD, E.M.; DANIELSEN, V. Dietary fibre for sows: effects of large amounts of soluble and insoluble fibres in the pregnancy period on the performance of sows during three reproductive cycles. Animal Science, v.68, p.355-362, 1998 .

YAN, T. et al. The digestion of dry matter and nonstarch polysaccharides from diets containing plain sugar-beet pulp or wheat straw by pregnant sows. British Society of Animal Science, v.61, p.305, 1995 . 